\title{
The role of continuity in residual-based variational multiscale modeling of turbulence
}

\author{
I. Akkerman - Y. Bazilevs • V. M. Calo • \\ T. J. R. Hughes - S. Hulshoff
}

Received: 10 May 2007 / Accepted: 16 May 2007 / Published online: 19 June 2007

(C) Springer-Verlag 2007

\begin{abstract}
This paper examines the role of continuity of the basis in the computation of turbulent flows. We compare standard finite elements and non-uniform rational B-splines (NURBS) discretizations that are employed in Isogeometric Analysis (Hughes et al. in Comput Methods Appl Mech Eng, 194:4135-4195, 2005). We make use of quadratic discretizations that are $C^{0}$-continuous across element boundaries in standard finite elements, and $C^{1}$-continuous in the case of NURBS. The variational multiscale residual-based method (Bazilevs in Isogeometric analysis of turbulence and fluidstructure interaction, PhD thesis, ICES, UT Austin, 2006; Bazilevs et al. in Comput Methods Appl Mech Eng, submitted, 2007; Calo in Residual-based multiscale turbulence modeling: finite volume simulation of bypass transition. $\mathrm{PhD}$ thesis, Department of Civil and Environmental Engineering, Stanford University, 2004; Hughes et al. in proceedings of the XXI international congress of theoretical and applied mechanics (IUTAM), Kluwer, 2004; Scovazzi in Multiscale methods in science and engineering, $\mathrm{PhD}$ thesis, Department of Mechanical Engineering, Stanford Universty, 2004) is employed as a turbulence modeling technique. We find that $C^{1}$-continuous discretizations outperform their $C^{0}$-continuous counterparts on a per-degree-of-freedom basis.
\end{abstract}

\footnotetext{
I. Akkerman · S. Hulshoff

Department of Aerospace Engineering,

Delft University of Technology,

Kluyverweg 1, 2629HS Delft,

The Netherlands

Y. Bazilevs $(\varangle)$ • V. M. Calo · T. J. R. Hughes

Institute for Computational Engineering and Sciences,

The University of Texas at Austin, 201 East 24th Street,

1 University Station C0200, Austin, TX 78712, USA

e-mail: bazily@ices.utexas.edu
}

We also find that the effect of continuity is greater for higher Reynolds number flows.

Keywords Incompressible flows - Finite elements . NURBS · Navier-Stokes equations · Boundary layers . Turbulent channel flows $\cdot$ Residual-based turbulence modeling · Isogeometric Analysis · Continuity of discretization - Variational multiscale formulation

\section{Introduction}

Due to the complicated multiscale nature of turbulence, numerical simulation of turbulent flows remains a significant computational challenge to this day. A community of researchers focused on understanding fundamental physical behavior of turbulence through numerical simulation typically makes use of very simple geometries and high-order spectral or compact finite difference methods (see, e.g., $[32,34])$. The underlying function spaces utilized in spectral methods are of high continuity $\left(C^{\infty}\right.$ in the cases of Fourier series and global polynomials). On the other hand, turbulent flows are also of great interest in general geometry industrial applications. These are typically computed using finite volume and finite element methods, which employ low-order approximation functions that are at most $C^{0}$-continuous.

Recently, Hughes et al. [25] introduced a new computational technique termed Isogeometric Analysis in an attempt to generalize and improve on the finite element method in the areas of geometry and solution representation, as well as mesh refinement. Isogeometric analysis is based on the geometric primitives of computer graphics and computer-aided design systems. The first instantiation of isogeometric analysis made use of non-uniform rational B-splines (NURBS, 
see $[16,36,37])$. NURBS-based isogeometric analysis was successfully applied to solve problems of vascular fluidstructure interaction in [5]. Accuracy of the new methodology for structural vibrations was shown to be superior to that of standard finite elements in [17]. A mathematical theory of NURBS-based isogeometric analysis was put forth in [4]. A distinguishing feature of isogeometric analysis is so-called $k$-refinement, in which the order of the functions is increased together with their continuity. As a result, isogeometric analysis allows for higher-order and higher-continuity discretizations on complex geometries, ${ }^{1}$ and may be thought of as bridging the gap existing between the procedures employed in the flow-physics and industrial-flow communities.

A variational multiscale, residual-based turbulence modeling framework has recently emerged as a new concept in large-eddy simulation (LES) of turbulent flows (see [2,6,12, $24,38])$. In this methodology one obtains an exact equation for the coarse, or resolved, scales and their explicit dependence on the fine, or unresolved, scales, and the modeling task amounts to accurately representing the fine scales in the coarse-scale equations. In this paper, inspired by the theory of stabilized methods, we employ simple algebraic models for the fine scales, which involve appropriately-scaled residuals of the underlying partial differential equations. For a summary of the early literature on stabilized methods see Brooks and Hughes [10]. Recent work on stabilized methods is presented in $[1,8,9,11,14,18-21,28,33,41-43]$.

Spectral studies of simple advective and diffusive model problems in [6] indicated better accuracy for the $C^{1}$-continuous case, especially in the upper part of the spectrum. NURBS-based isogeometric analysis, in conjunction with the multiscale approach, was applied to turbulent flow computations in [2]. Preliminary results indicated better accuracy for higher-order and higher-continuity discretizations. It was noted in [2] that when going from a $C^{0}$-continuous linear to a $C^{1}$-continuous quadratic $\mathrm{B}$-spline basis an increase in solution accuracy was obtained. The question this raises is whether it was the order or continuity of the basis functions that led to superior accuracy. It is the primary goal of this paper to answer this question. It should be noted that good accuracy of B-spline discretizations for wall-bounded turbulent flows was also noted in [29-31,40].

The variational multiscale formulation employed in this study utilized the "advective form" of the convection term rather than the integrated by parts "conservative form". The advective form is often employed in finite element flow codes, perhaps more so than the conservative form. In earlier works

\footnotetext{
${ }^{1}$ It is important to note that the highest possible order of continuity of the solution space in a NURBS-based isogeometric analysis is limited to the continuity of the basis used in the definition of the geometrical domain of interest. Pure $k$-refinement with maximal smoothness is only attainable in simple geometries.
}

on residual-based approaches $[2,6,12,24]$, the conservative form was employed.

The paper is organized as follows. In Sect.2, we state the weak formulation of the incompressible Navier-Stokes equations at the continuous level. In Sect. 3, we give the semidiscrete, residual-based variational multiscale formulation of the incompressible Navier-Stokes equations in advective form. In Sect. 4, we present numerical results for equilibrium turbulent channel flows at friction-velocity Reynolds numbers 180 and 590. We compare $C^{0}$ - and $C^{1}$-continuous quadratic elements and assess accuracy on the basis of the number of degrees of freedom. High-fidelity, direct numerical simulation (DNS) results of [35] are used as reference solutions. In Sect. 5, we draw conclusions.

\section{Incompressible Navier-Stokes equations}

We begin by considering a weak formulation of the incompressible Navier-Stokes equations. Let $\mathcal{V}$ denote the trial solution and weighting function spaces, which are assumed to be the same. We also assume $\boldsymbol{u}=\mathbf{0}$ on $\Gamma$ and $\int_{\Omega} p(t) d \Omega=0$ for all $t \in] 0, T[$. The variational formulation is stated as follows: Find $\boldsymbol{U}=\{\boldsymbol{u}, p\} \in \mathcal{V}$ such that $\forall \boldsymbol{W}=\{\boldsymbol{w}, q\} \in \mathcal{V}$,

$B(\boldsymbol{W}, \boldsymbol{U})=(\boldsymbol{W}, \boldsymbol{F})$

where

$$
\begin{aligned}
B(\boldsymbol{W}, \boldsymbol{U})= & \left(\boldsymbol{w}, \frac{\partial \boldsymbol{u}}{\partial t}\right)_{\Omega}-(\nabla \boldsymbol{w}, \boldsymbol{u} \otimes \boldsymbol{u})_{\Omega}+(q, \nabla \cdot \boldsymbol{u})_{\Omega} \\
& -(\nabla \cdot \boldsymbol{w}, p)_{\Omega}+\left(\nabla^{s} \boldsymbol{w}, 2 v \nabla^{s} \boldsymbol{u}\right)_{\Omega},
\end{aligned}
$$

$(\boldsymbol{W}, \boldsymbol{F})=(\boldsymbol{w}, \boldsymbol{f})_{\Omega}$,

and

$\nabla^{s} \boldsymbol{u}=\frac{1}{2}\left(\nabla \boldsymbol{u}+\nabla \boldsymbol{u}^{T}\right)$.

$f$ is the force (per unit mass), $v$ is the kinematic viscosity and $p$ is the pressure divided by the density.

Variational equations (1)-(3) imply weak satisfaction of the linear momentum equations and incompressibility constraint, namely

$$
\begin{aligned}
& \frac{\partial \boldsymbol{u}}{\partial t}+\nabla \cdot(\boldsymbol{u} \otimes \boldsymbol{u})+\nabla p-\nabla \cdot\left(2 v \nabla^{s} \boldsymbol{u}\right)-\boldsymbol{f}=\mathbf{0} \quad \text { in } \Omega \\
& \nabla \cdot \boldsymbol{u}=0 \quad \text { in } \Omega .
\end{aligned}
$$

Note that one may use the incompressibility constraint to simplify the momentum equation as

$$
\frac{\partial \boldsymbol{u}}{\partial t}+\boldsymbol{u} \cdot \nabla \boldsymbol{u}+\nabla p-v \Delta \boldsymbol{u}-\boldsymbol{f}=\mathbf{0} \text { in } \Omega .
$$




\section{Multiscale residual-based formulation of the incompressible Navier-Stokes equations employing the advective form}

We consider a direct-sum decomposition of $\mathcal{V}$ into "coarsescale" and "fine-scale" subspaces, $\mathcal{V}^{h}$ and $\mathcal{V}^{\prime}$, respectively,

$\mathcal{V}=\mathcal{V}^{h} \oplus \mathcal{V}^{\prime}$

$\mathcal{V}^{h}$ is assumed to be a finite-dimensional space, which will be identified later with the space of functions with which we actually compute. One obtains a unique decomposition in (8) with the aid of a linear projection operator $\mathcal{P}$, that gives $\boldsymbol{U}^{h}=\mathcal{P} \boldsymbol{U} \in \mathcal{V}^{h}$ and $\boldsymbol{U}^{\prime}=(\mathcal{I}-\mathcal{P}) \boldsymbol{U} \in \mathcal{V}^{\prime}$ from a given $\boldsymbol{U} \in \mathcal{V}$ (see [23] for details).

By restricting the weighting space to $\mathcal{V}^{h}$ in (1) and employing the direct-sum decomposition (8) for the solution space, we obtain the equation system for the large scales, namely: Find $\boldsymbol{U}^{h}=\left\{\boldsymbol{u}^{h}, p^{h}\right\} \in \mathcal{V}^{h}$ such that $\forall \boldsymbol{W}^{h}=\left\{\boldsymbol{w}^{h}, q^{h}\right\} \in \mathcal{V}^{h}$,

$B\left(\boldsymbol{W}^{h}, \boldsymbol{U}^{h}+\boldsymbol{U}^{\prime}\right)=\left(\boldsymbol{W}^{h}, \boldsymbol{F}\right)$.

(9) indicates precisely the manner in which the large scales depend on $\boldsymbol{U}^{\prime}=\left\{\boldsymbol{u}^{\prime}, p^{\prime}\right\}$.

Combining (2) and (9), we obtain: Find $\boldsymbol{U}^{h} \in \mathcal{V}^{h}$, such that $\forall W^{h} \in \mathcal{V}^{h}$,

$$
\begin{aligned}
& \left(\boldsymbol{w}^{h}, \frac{\partial \boldsymbol{u}^{h}}{\partial t}\right)_{\Omega}-\left(\nabla \boldsymbol{w}^{h}, \boldsymbol{u}^{h} \otimes \boldsymbol{u}^{h}\right)_{\Omega}+\left(q^{h}, \nabla \cdot \boldsymbol{u}^{h}\right)_{\Omega} \\
& -\left(\nabla \cdot \boldsymbol{w}^{h}, p^{h}\right)_{\Omega}+\left(\nabla^{s} \boldsymbol{w}^{h}, 2 v \nabla^{s} \boldsymbol{u}^{h}\right)_{\Omega}-\left(\boldsymbol{w}^{h}, \boldsymbol{f}\right)_{\Omega} \\
& +\left(\boldsymbol{w}^{h}, \frac{\partial \boldsymbol{u}^{\prime}}{\partial t}\right)_{\Omega}-\left(\nabla \boldsymbol{w}^{h}, \boldsymbol{u}^{h} \otimes \boldsymbol{u}^{\prime}\right)_{\Omega}-\left(\nabla \boldsymbol{w}^{h}, \boldsymbol{u}^{\prime} \otimes \boldsymbol{u}^{h}\right)_{\Omega} \\
& -\left(\nabla \boldsymbol{w}^{h}, \boldsymbol{u}^{\prime} \otimes \boldsymbol{u}^{\prime}\right)_{\Omega}+\left(q^{h}, \nabla \cdot \boldsymbol{u}^{\prime}\right)_{\Omega} \\
& -\left(\nabla \cdot \boldsymbol{w}^{h}, p^{\prime}\right)_{\Omega}+\left(\nabla^{s} \boldsymbol{w}^{h}, 2 v \nabla^{s} \boldsymbol{u}^{\prime}\right)_{\Omega}=0
\end{aligned}
$$

For purposes of modeling the fine scales we make the simplifying assumption $\left(\boldsymbol{w}^{h}, \frac{\partial \boldsymbol{u}^{\prime}}{\partial t}\right)_{\Omega}=0$. We note, however, that it has been shown in [15] that it is beneficial to incorporate this effect in modeling the fine scales. The term $\left(\nabla^{s} \boldsymbol{w}^{h}, 2 \nabla^{s} \boldsymbol{u}^{\prime}\right)_{\Omega}$ may be omitted by selecting a projector that enforces the orthogonality of the coarse and fine scales in the semi-norm induced by this term (see, e.g., [2]).

We turn our attention to the convective terms in (10). Assuming incompressibility of the velocity field, namely, $\nabla \cdot\left(\boldsymbol{u}^{h}+\boldsymbol{u}^{\prime}\right)=0$, we compute:

$$
\begin{aligned}
- & \left(\nabla \boldsymbol{w}^{h}, \boldsymbol{u}^{h} \otimes \boldsymbol{u}^{h}\right)_{\Omega}-\left(\nabla \boldsymbol{w}^{h}, \boldsymbol{u}^{h} \otimes \boldsymbol{u}^{\prime}\right)_{\Omega} \\
- & \left(\nabla \boldsymbol{w}^{h}, \boldsymbol{u}^{\prime} \otimes \boldsymbol{u}^{h}\right)_{\Omega}-\left(\nabla \boldsymbol{w}^{h}, \boldsymbol{u}^{\prime} \otimes \boldsymbol{u}^{\prime}\right)_{\Omega} \\
= & -\left(\nabla \boldsymbol{w}^{h}, \boldsymbol{u}^{h} \otimes\left(\boldsymbol{u}^{h}+\boldsymbol{u}^{\prime}\right)\right)_{\Omega} \\
& -\left(\nabla \boldsymbol{w}^{h}, \boldsymbol{u}^{\prime} \otimes \boldsymbol{u}^{h}\right)_{\Omega}-\left(\nabla \boldsymbol{w}^{h}, \boldsymbol{u}^{\prime} \otimes \boldsymbol{u}^{\prime}\right)_{\Omega} \\
= & \left(\boldsymbol{w}^{h},\left(\boldsymbol{u}^{h}+\boldsymbol{u}^{\prime}\right) \cdot \nabla \boldsymbol{u}^{h}\right)_{\Omega} \\
& -\left(\nabla \boldsymbol{w}^{h}, \boldsymbol{u}^{\prime} \otimes \boldsymbol{u}^{h}\right)_{\Omega}-\left(\nabla \boldsymbol{w}^{h}, \boldsymbol{u}^{\prime} \otimes \boldsymbol{u}^{\prime}\right)_{\Omega}
\end{aligned}
$$

At this point we assume that $\Omega$ is partitioned into a set of subdomains, such as finite elements or NURBS elements, and on this partition we have a finite dimensional space of functions with local support that forms our approximation space for $\boldsymbol{U}^{h}$ and $\boldsymbol{W}^{h}$. Let $\boldsymbol{x}=\left\{x_{i}\right\}_{i=1}^{d}$, denote the coordinates of element $K$ in physical space, and let $\boldsymbol{\xi}=\left\{\xi_{i}\right\}_{i=1}^{d}$, denote the coordinates of element $\hat{K}$ in parametric space. Let $\boldsymbol{x}=\boldsymbol{x}(\boldsymbol{\xi}): \hat{K} \rightarrow K$ be a continuously differentiable map with a continuously differentiable inverse.

We model the fine scales as in [6]:

$\boldsymbol{U}^{\prime} \approx-\boldsymbol{\tau} \boldsymbol{R}\left(\boldsymbol{U}^{h}\right)$,

where $\tau$ is a $4 \times 4$ matrix (in three spatial dimensions) and $\boldsymbol{R}\left(\boldsymbol{U}^{h}\right)$ is a $4 \times 1$ vector that collects momentum and continuity residuals of the Navier-Stokes equations,

$\boldsymbol{R}\left(\boldsymbol{U}^{h}\right)=\left\{\boldsymbol{r}_{M}^{T}\left(\boldsymbol{u}^{h}, p^{h}\right), r_{C}\left(\boldsymbol{u}^{h}\right)\right\}^{T}$,

in which

$\boldsymbol{r}_{M}\left(\boldsymbol{u}^{h}, p^{h}\right)=\frac{\partial \boldsymbol{u}^{h}}{\partial t}+\boldsymbol{u}^{h} \cdot \nabla \boldsymbol{u}^{h}+\nabla p^{h}-v \Delta \boldsymbol{u}^{h}-\boldsymbol{f}$,

$r_{C}\left(\boldsymbol{u}^{h}\right)=\nabla \cdot \boldsymbol{u}^{h}$

We define $\boldsymbol{\tau}$ as follows:

$\boldsymbol{\tau}=\operatorname{diag}\left(\tau_{M}, \tau_{M}, \tau_{M}, \tau_{C}\right)$,

where

$\tau_{M}=\left(\frac{4}{\Delta t^{2}}+\boldsymbol{u}^{h} \cdot \boldsymbol{G} \boldsymbol{u}^{h}+C_{I} v^{2} \boldsymbol{G}: \boldsymbol{G}\right)^{-1 / 2}$,

$\tau_{C}=\left(g \cdot \tau_{M} g\right)^{-1}$,

with $\boldsymbol{G}$ a second rank metric tensor

$\boldsymbol{G}=\frac{\partial \boldsymbol{\xi}^{T}}{\partial \boldsymbol{x}} \frac{\partial \boldsymbol{\xi}}{\partial \boldsymbol{x}}$, 
and $\boldsymbol{g}$ a vector obtained from the column sums of $\frac{\partial \boldsymbol{\xi}}{\partial \boldsymbol{x}}$,

$\boldsymbol{g}=\left\{g_{i}\right\}$

$g_{i}=\sum_{j=1}^{d}\left(\frac{\partial \xi}{\partial \boldsymbol{x}}\right)_{j i}$.

The definition of $\tau_{M}$ in (17) is inspired by the theory of stabilized methods for advection-diffusion-reaction systems (see, e.g., Shakib et al. [39], Hughes and Mallet [22]). The definition of $\tau_{C}$ comes from the small-scale Shur complement operator for the pressure (see [2] for a details). In the definition of $\tau_{M}$ (17), $C_{I}$ is a positive constant, independent of the mesh size, that derives from the element-wise inverse estimate (see, e.g., [27]).

Combining equations (10)-(12), we obtain our discrete formulation: Find $\boldsymbol{U}^{h} \in \mathcal{V}^{h}$, such that $\forall \boldsymbol{W}^{h} \in \mathcal{V}^{h}$,

$$
\begin{gathered}
\left(\boldsymbol{w}^{h}, \frac{\partial \boldsymbol{u}^{h}}{\partial t}\right)_{\Omega}+\left(\boldsymbol{w}^{h},\left(\boldsymbol{u}^{h}-\tau_{M} \boldsymbol{r}_{M}\right) \cdot \nabla \boldsymbol{u}^{h}\right)_{\Omega} \\
+\left(q^{h}, \nabla \cdot \boldsymbol{u}^{h}\right)_{\Omega}-\left(\nabla \cdot \boldsymbol{w}^{h}, p^{h}\right)_{\Omega}
\end{gathered}
$$

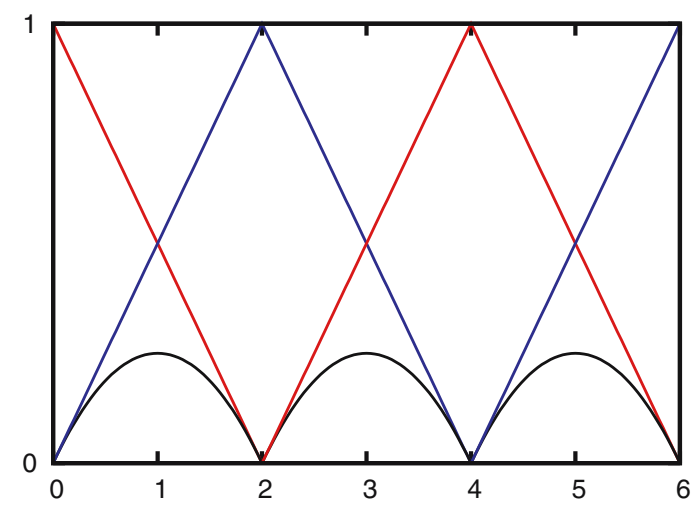

(a) $C^{0}$-continuous quadratic elements

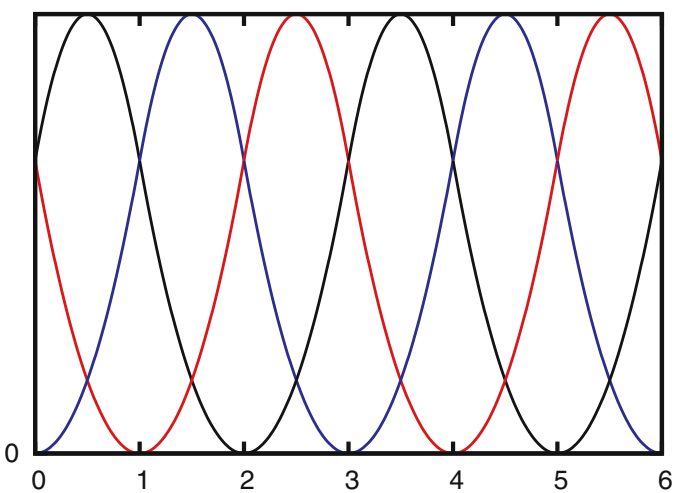

(b) $C^{1}$-continuous quadratic NURBS

Fig. 1 Basis functions employed in homogeneous directions. Basis functions are shown in a univariate setting. Three-dimensional basis functions are obtained by taking tensor products of one-dimensional basis functions. The meshes are stretched in the wall-normal direction

$$
\begin{aligned}
& +\left(\nabla^{s} \boldsymbol{w}^{h}, 2 v \nabla^{s} \boldsymbol{u}^{h}\right)_{\Omega}-\left(\boldsymbol{w}^{h}, \boldsymbol{f}\right)_{\Omega} \\
& +\left(\boldsymbol{u}^{h} \cdot \nabla \boldsymbol{w}^{h}+\nabla q^{h}, \tau_{M} \boldsymbol{r}_{M}\right)_{\Omega}+\left(\nabla \cdot \boldsymbol{w}^{h}, \tau_{C} \nabla \cdot \boldsymbol{u}^{h}\right)_{\Omega} \\
& -\left(\nabla \boldsymbol{w}^{h}, \tau_{M} \boldsymbol{r}_{M} \otimes \tau_{M} \boldsymbol{r}_{M}\right)_{\Omega}=0 .
\end{aligned}
$$

\section{Numerical experiments for turbulent channel flow}

\subsection{Problem setup}

To examine the effects of continuity, we compute turbulent channel flows at Reynolds numbers $R e_{\tau}=180$ and $R e_{\tau}=$ 590 , based on the friction velocity and the channel half width. To assess the accuracy of the calculations, we compare with the DNS of [35].

The problem setup is as follows. The computational domain is a rectangular box, and the flow is driven by a

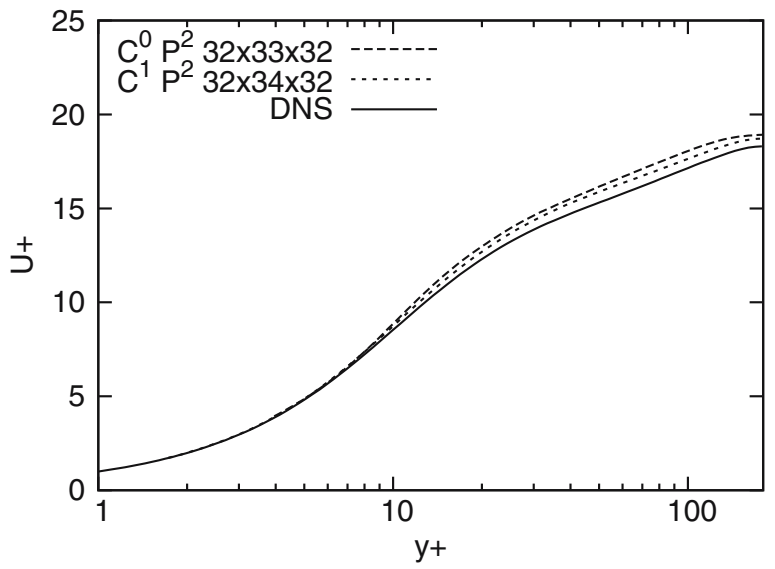

(a) Mean stream-wise velocity

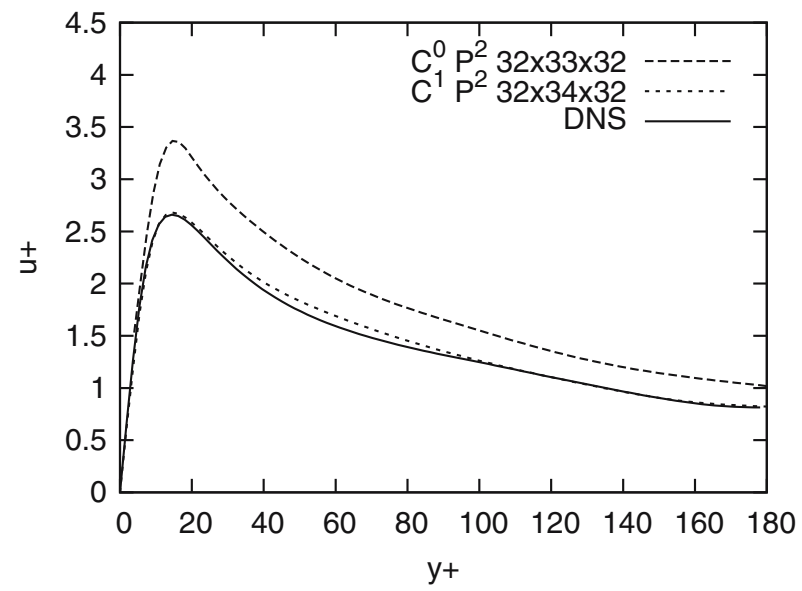

(b) Stream-wise velocity fluctuations

Fig. 2 Turbulent channel flow at $R e_{\tau}=180$ computed on a $32^{3}$ element mesh. Comparison of $C^{0}$ - versus $C^{1}$-continuous discretizations 


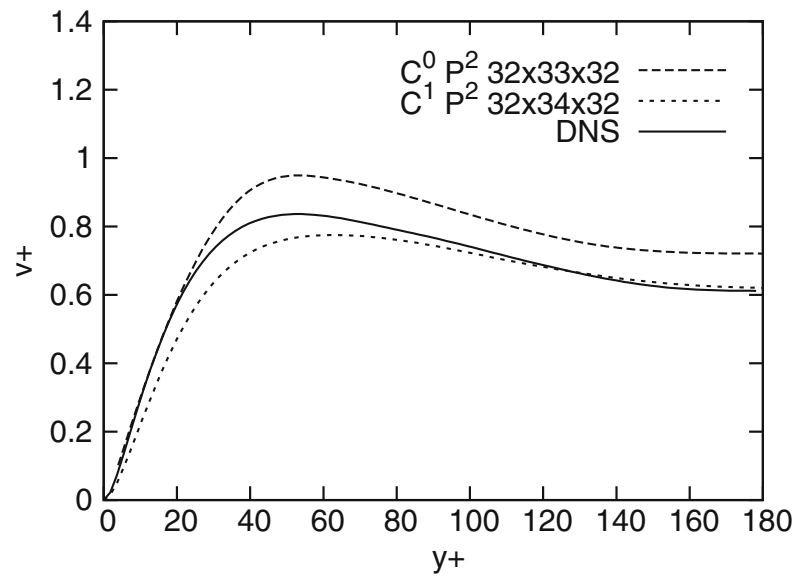

(a) Wall-normal velocity fluctuations

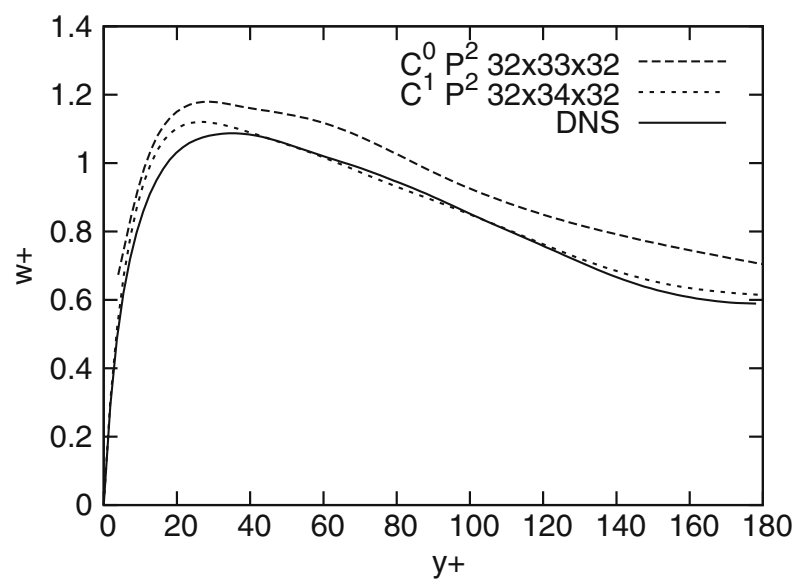

(b) Span-wise velocity fluctuations

Fig. 3 Turbulent channel flow at $R e_{\tau}=180$ computed on a $32^{3}$ element mesh. Comparison of $C^{0}$ - versus $C^{1}$-continuous discretizations

constant pressure gradient in the stream-wise direction. Periodic boundary conditions are imposed in the stream-wise and span-wise directions, commonly referred to as homogeneous directions. A no-slip boundary condition is applied at the walls. The no-slip boundary condition is enforced strongly, that is, the discrete velocity is set to zero at the walls. An alternative approach is to enforce Dirichlet boundary conditions weakly. This is accomplished by appropriately augmenting the semi-discrete equations (21) by terms that enforce the no-slip condition weakly (see $[3,7]$ for details). Although the latter approach was shown to be superior to the former, we did not employ it in the computations presented herein.

We employ quadratic finite elements that are $C^{0}$-continuous, and quadratic NURBS elements that are $C^{1}$-continuous across element interfaces. The basis functions utilized in our computations are shown in Fig. 1.

The semi-discrete equations (21) are advanced in time using the generalized- $\alpha$ method $[13,26]$. We use meshes that are stretched in the wall-normal direction according to a

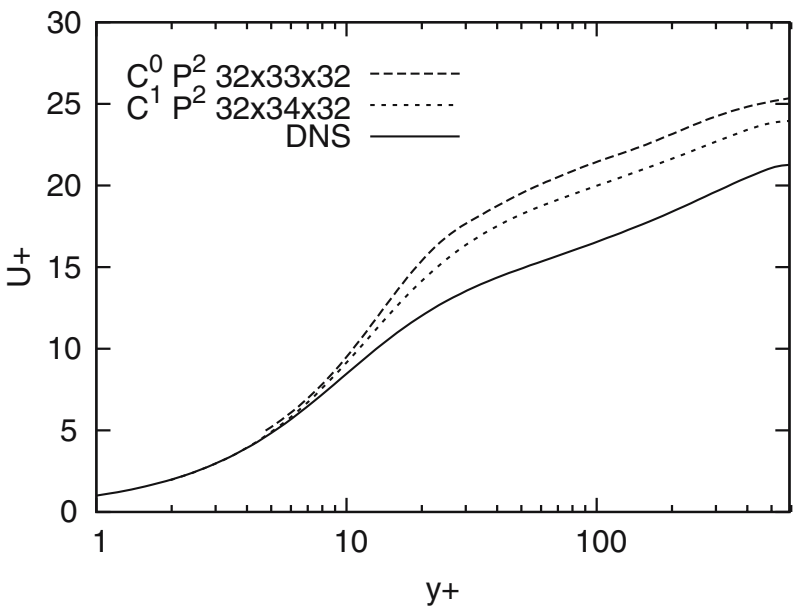

(a) Mean stream-wise velocity

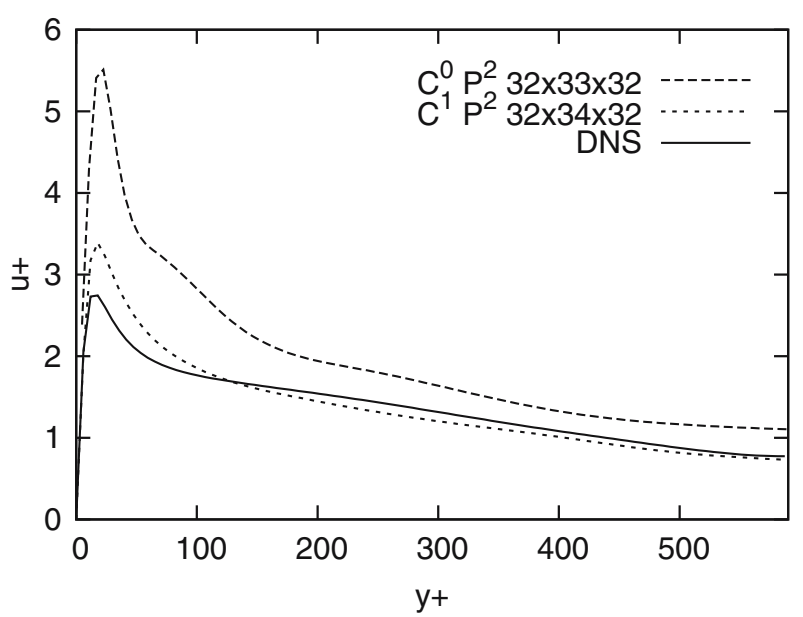

(b) Stream-wise velocity fluctuations

Fig. 4 Turbulent channel flow at $R e_{\tau}=590$ computed on a $32^{3}$ element mesh. Comparison of $C^{0}$ - versus $C^{1}$-continuous discretizations

hyperbolic function to cluster points near the wall. Moreover, in the definition of $\tau_{M}$ (17) we set $C_{I}$ to 36 .

Numerical results for all cases are reported in the form of statistics of the mean velocity and root-mean-square velocity fluctuations. The statistics were computed by sampling the velocity field at the mesh knots and averaging the solution in time as well as in the homogeneous directions. The meshes were chosen such that the number of degrees of freedom for both quadratic discretizations are approximately the same. All computational results are presented in non-dimensional wall units.

\subsection{Turbulent channel flow at $R e_{\tau}=180$}

The domain size is $2 \pi, 2$, and $4 / 3 \pi$ in the stream-wise, wallnormal, and span-wise directions, respectively. The corresponding DNS computation was carried out on a domain of 


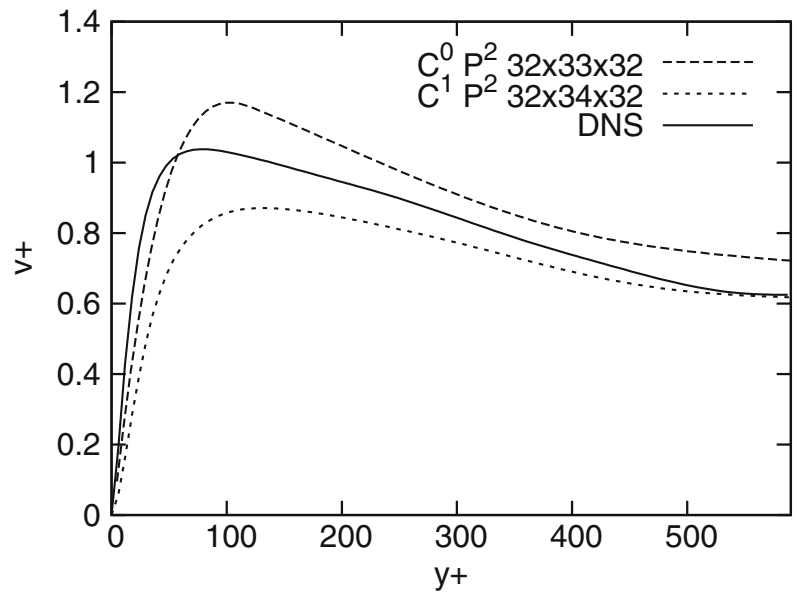

(a) Wall-normal velocity fluctuations

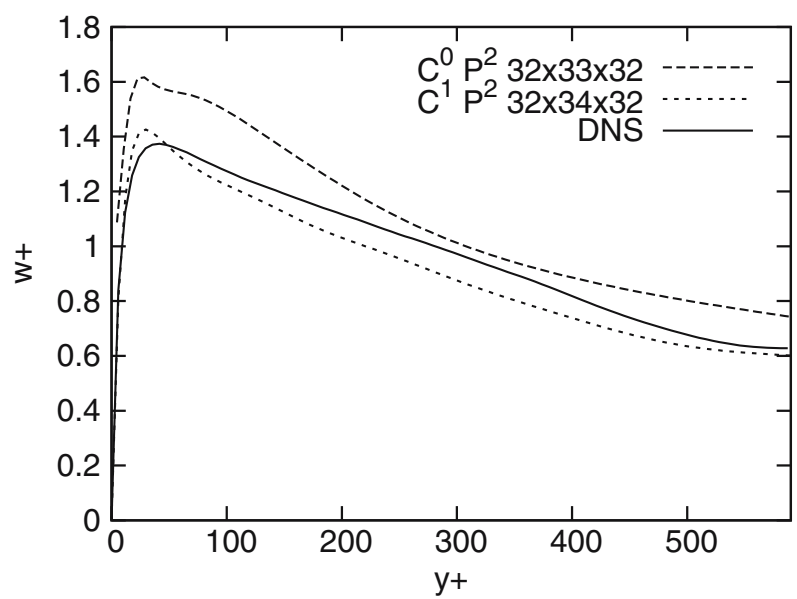

(b) Span-wise velocity fluctuations

Fig. 5 Turbulent channel flow at $R e_{\tau}=590$ computed on a $32^{3}$ element mesh. Comparison of $C^{0}$ - versus $C^{1}$-continuous discretizations

the same size with $128 \times 129 \times 128$ spectral functions in the stream-wise, wall-normal and span-wise direction, respectively.

We carried out computations employing $C^{0}$ - and $C^{1}$-continuous quadratic discretizations keeping the number of degrees of freedom nearly the same in both cases. For the $C^{0}$ case we used a mesh of $16^{3}$ elements, which gave $32 \times 33 \times 32$ basis functions in our discrete space, whereas for the $C^{1}$ case we employed a mesh of $32^{3}$ elements, which led to a discrete space comprised of $32 \times 34 \times 32$ basis functions. (The open knot vector construction is responsible for the extra basis function in the wall-normal direction; see Hughes et al. [25].)

Figure 2 illustrates that the mean flow obtained with the $C^{1}$-continuous discretization is slightly more accurate. However, both discretizations show good agreement with the DNS result. On the other hand, the fluctuations are significantly better in the case of $C^{1}$ quadratics (see Figs. 2, 3).

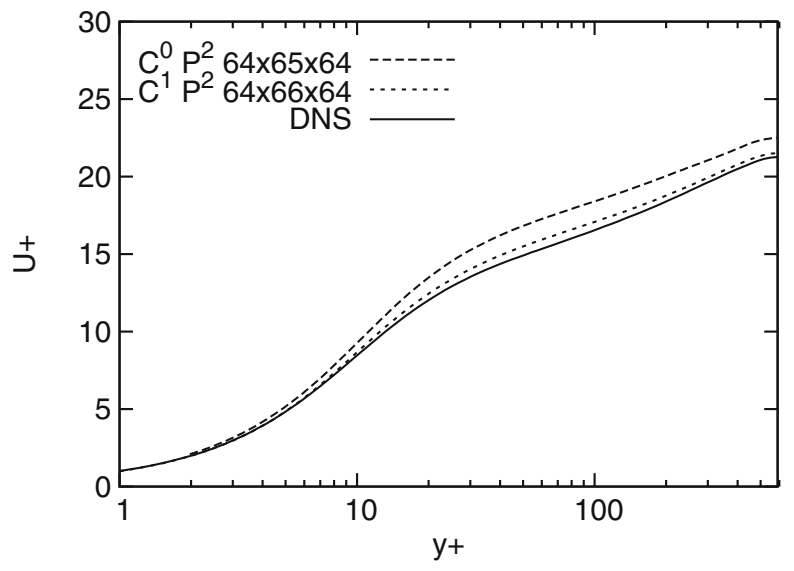

(a) Mean stream-wise velocity

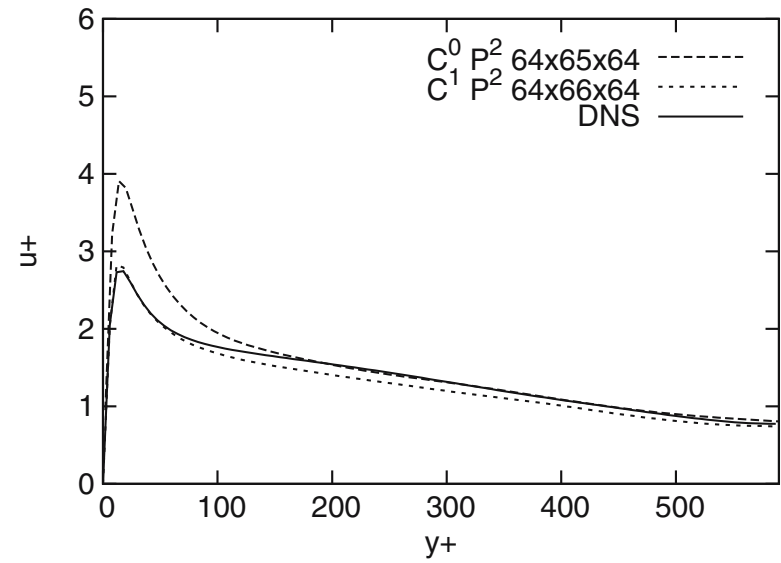

(b) Stream-wise velocity fluctuations

Fig. 6 Turbulent channel flow at $R e_{\tau}=590$ computed on a $64^{3}$ element mesh. Comparison of $C^{0}$ - versus $C^{1}$-continuous discretizations

\subsection{Turbulent channel flow at $R e_{\tau}=590$}

We also performed turbulent channel flow computations for $R e_{\tau}=590$ to examine the effects of the continuity as the Reynolds number of the flow is increased. For this simulation the domain size is $2 \pi, 2$, and $\pi$ in the stream-wise, wall-normal and span-wise directions, respectively. The corresponding DNS used the same domain size with a resolution of $384 \times 257 \times 384$ spectral functions in the stream-wise, wall-normal and span-wise directions.

As in the $R e_{\tau}=180$ case, we present results comparing quadratic discretizations that are $C^{0}$ - and $C^{1}$-continuous while keeping the number of degrees of freedom nearly the same. Figures 4, 5 show the results obtained using a $C^{0}$ mesh of $32 \times 33 \times 32$ basis functions and a $C^{1}$ mesh of $32 \times 34 \times 32$ basis functions. The difference in the number of degrees of freedom is, as mentioned previously, attributable to the open knot vector construction for the NURBS basis. These meshes are considered coarse for this simulation, which is manifested by the fact that the mean stream-wise velocity is 


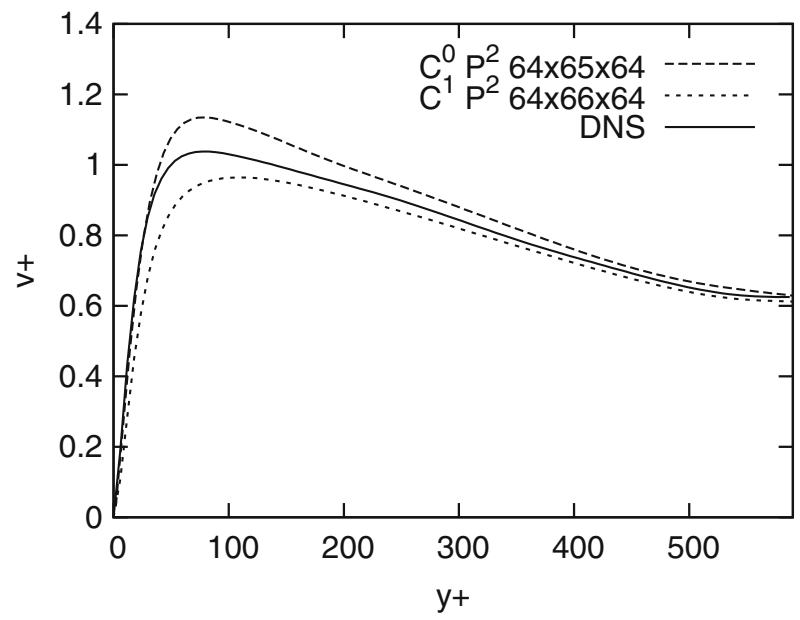

(a) Wall-normal velocity fluctuations

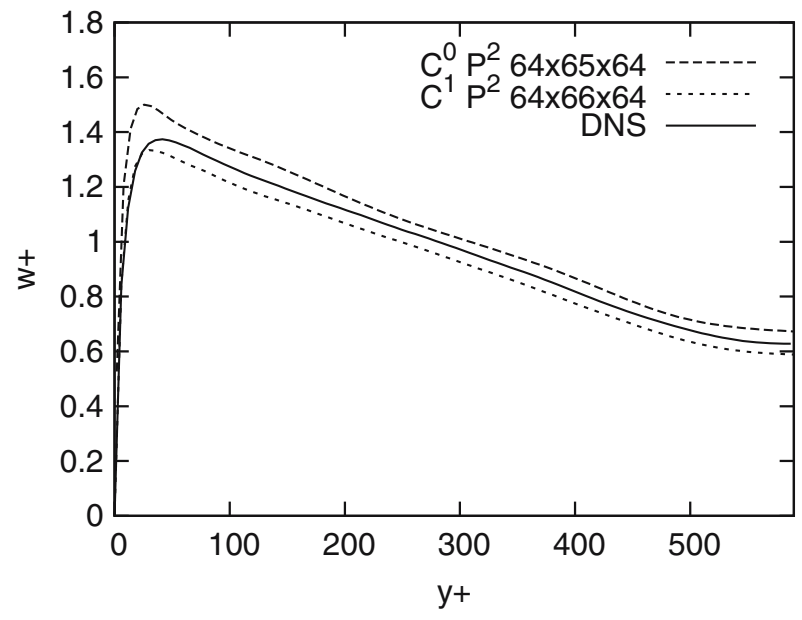

(b) Span-wise velocity fluctuations

Fig. 7 Turbulent channel flow at $R e_{\tau}=590$ computed on a $64^{3}$ element mesh. Comparison of $C^{0}$ - versus $C^{1}$-continuous discretizations

over-predicted by both discretizations, the $C^{1}$ discretization being more accurate (see Fig. 4). As in the $R e_{\tau}=180$ case, the velocity fluctuations are significantly more accurate for $C^{1}$ quadratic NURBS, as shown in Figs. 4, 5.

We performed the same comparison study on $h$-refined meshes, resulting in $64 \times 65 \times 64$ functions for the $C^{0}$ basis and $64 \times 66 \times 64$ functions for the $C^{1}$ basis. Results are presented in Figs. 6, 7. Both the mean flow and fluctuations are more accurate in the case of $C^{1}$ NURBS, for which the mean steam-wise velocity is very close to the DNS result. From the above results one may infer that solution accuracy depends more critically on the continuity of the discretization for flows at higher Reynolds number.

\section{Conclusions}

In this work, we presented a residual-based, variational multiscale model of turbulence that is based on the advective form of the convection term, a popular choice in finite element flow codes. We compared turbulent channel flow results using $C^{0}$ - and $C^{1}$-continuous quadratic discretizations. Using a $C^{1}$-continuous quadratic basis yields more accurate mean flow and fluctuating quantities than $C^{0}$-continuous quadratic basis functions. We conclude that smooth NURBS basis functions have advantages over $C^{0}$-continuous finite elements in turbulent flow calculations as anticipated in [6].

Acknowledgements This research was supported by Office of Naval Research Contract N00014-03-0263, Dr. Luise Couchman, contract monitor, and Sandia National Laboratories under contract number 114166. I. Akkerman was supported by the Dutch Technology Foundation STW, applied science division of NWO, and the Technology Program of the Ministry of Economic Affairs. Y. Bazilevs was partially supported by the J.T. Oden ICES Postdoctoral Fellowship at the Institute for Computational Engineering and Sciences (ICES). This support is gratefully acknowledged.

\section{References}

1. Akin JE, Tezduyar TE (2004) Calculation of the advective limit of the SUPG stabilization parameter for linear and higher-order elements. Comput Methods Appl Mech Eng 193:1909-1922

2. Bazilevs Y (2006) Isogeometric analysis of turbulence and fluidstructure interaction. PhD thesis, ICES, UT Austin

3. Bazilevs Y, Hughes TJR (2007) Weak imposition of Dirichlet boundary conditions in fluid mechanics. Comput Fluids 36:12-26

4. Bazilevs Y, Beirao da Veiga L, Cottrell JA, Hughes TJR, Sangalli G (2006) Isogeometric analysis: approximation, stability and error estimates for $h$-refined meshes. Math Models Methods Appl Sci 16:1031-1090

5. Bazilevs Y, Calo VM, Zhang Y, Hughes TJR (2006) Isogeometric fluid-structure interaction analysis with applications to arterial blood flow. Comput Mech 38:310-322

6. Bazilevs Y, Calo VM, Cottrell JA, Hughes TJR, Reali A, Scovazzi G (2007) Variational multiscale residual-based turbulence modeling for large eddy simulation of incompressible flows. Comput Methods Appl Mech Eng. Available as ICES Report 07-15, UT Austin (submitted)

7. Bazilevs Y, Michler C, Calo VM, Hughes TJR (2007) Weak Dirichlet boundary conditions for wall-bounded turbulent flows. Comput Methods Appl Mech Eng (accepted for publication)

8. Bischoff M, Bletzinger K-U (2004) Improving stability and accuracy of Reissner-Mindlin plate finite elements via algebraic subgrid scale stabilization. Comput Methods Appl Mech Eng 193:14911516

9. Bochev PB, Gunzburger MD, Shadid JN (2004) On inf-sup stabilized finite element methods for transient problems. Comput Methods Appl Mech Eng 193:1471-1489

10. Brooks AN, Hughes TJR (1982) Streamline upwind/PetrovGalerkin formulations for convection dominated flows with particular emphasis on the incompressible Navier-Stokes equations. Comput Methods Appl Mech Eng 32:199-259

11. Burman E, Hansbo P (2004) Edge stabilization for Galerkin approximations of convection-diffusion-reaction problems. Comput Methods Appl Mech Eng 193:1437-1453

12. Calo VM (2004) Residual-based multiscale turbulence modeling: finite volume simulation of bypass transistion. $\mathrm{PhD}$ thesis, Department of Civil and Environmental Engineering, Stanford University

13. Chung J, Hulbert GM (1993) A time integration algorithm for structural dynamics with improved numerical dissipation: the generalized- $\alpha$ method. J Appl Mech 60:371-375 
14. Codina R, Soto O (2004) Approximation of the incompressible Navier-Stokes equations using orthogonal subscale stabilization and pressure segregation on anisotropic finite element meshes. Comput Methods Appl Mech Eng 193:1403-1419

15. Codina R, Principe J, Guasch O, Badia S (2007) Time dependent subscales in the stabilized finite element approximation of incompressible flow problems. Comput Methods Appl Mech Eng (accepted for publication)

16. Cohen E, Riesenfeld R, Elber G (2001) Geometric modeling with splines. An introduction. A K Peters Ltd, Wellesley

17. Cottrell JA, Reali A, Bazilevs Y, Hughes TJR (2006) Isogeometric analysis of structural vibrations. Comput Methods Appl Mech Eng 195:5257-5297

18. Coutinho ALGA, Diaz CM, Alvez JLD, Landau L, Loula AFD, Malta SMC, Castro RGS, Garcia ELM (2004) Stabilized methods and post-processing techniques for miscible displacements. Comput Methods Appl Mech Eng 193:1421-1436

19. Gravemeier V, Wall WA, Ramm E (2004) A three-level finite element method for the instationary incompressible NavierStokes equations. Comput Methods Appl Mech Eng 193:13231366

20. Harari I (2004) Stability of semidiscrete formulations for parabolic problems at small time steps. Comput Methods Appl Mech Eng 193:1491-1516

21. Hauke G, Valiño L (2004) Computing reactive flows with a field Monte Carlo formulation and multi-scale methods. Comput Methods Appl Mech Eng 193:1455-1470

22. Hughes TJR, Mallet M (1986) A new finite element formulation for fluid dynamics: III. The generalized streamline operator for multidimensional advective-diffusive systems. Comput Methods Appl Mech Eng 58:305-328

23. Hughes TJR, Sangalli G (2006) Variational multiscale analysis: the fine-scale Green's function, projection, optimization, localization, and stabilized methods. SIAM J Numer Anal (accepted). Available as ICES Report 05-46, UT Austin

24. Hughes TJR, Calo VM, Scovazzi G (2004) Variational and multiscale methods in turbulence. In: Gutkowski W, Kowalewski TA (eds) Proceedings of the XXI international congress of theoretical and applied mechanics (IUTAM). Kluwer, Dordrecht

25. Hughes TJR, Cottrell JA, Bazilevs Y (2005) Isogeometric analysis: CAD, finite elements, NURBS, exact geometry, and mesh refinement. Comput Methods Appl Mech Eng 194:4135-4195

26. Jansen KE, Whiting CH, Hulbert GM (1999) A generalized- $\alpha$ method for integrating the filtered Navier-Stokes equations with a stabilized finite element method. Comput Methods Appl Mech Eng 190:305-319
27. Johnson C (1987) Numerical solution of partial differential equations by the finite element method. Cambridge University Press, Sweden

28. Koobus B, Farhat C (2004) A variational multiscale method for the large eddy simulation of compressible turbulent flows on unstructured meshes-application to vortex shedding. Comput Methods Appl Mech Eng 193:1367-1383

29. Kravchenko AG, Moin P, Moser R (1996) Zonal embedded grids for numerical simulation of wall-bounded turbulent flows. J Comput Phys 127:412-423

30. Kravchenko AG, Moin P, Shariff K (1999) B-spline method and zonal grids for simulation of complex turbulent flows. J Comput Phys 151:757-789

31. Kwok WY, Moser RD, Jiménez J (2001) A critical evaluation of the resolution properties of B-spline and compact finite difference methods. J Comput Phys 174:510-551

32. Lele SK (1992) Compact finite difference schemes with spectrallike resolution. J Comput Phys 103:16-42

33. Masud A, Khurram RA (2004) A multiscale/stabilized finite element method for the advection-diffusion equation. Comput Methods Appl Mech Eng 193:1997-2018

34. Moin P (2001) Fundamentals of engineering numerical analysis. Cambridge University Press, London

35. Moser R, Kim J, Mansour R (1999) DNS of turbulent channel flow up to $R e=590$. Phys Fluids 11:943-945

36. Piegl L, Tiller W (1997) The NURBS book (Monographs in visual communication), 2nd edn. Springer, New York

37. Rogers DF (2001) An introduction to NURBS with historical perspective. Academic, San Diego

38. Scovazzi G (2004) Multiscale methods in science and engineering. $\mathrm{PhD}$ thesis, Department of Mechanical Engineering, Stanford Universty. Available at http://www.cs.sandia.gov/ gscovaz

39. Shakib F, Hughes TJR, Johan Z (1991) A new finite element formulation for computational fluid dynamics: $\mathrm{X}$. The compressible Euler and Navier-Stokes equations. Comput Methods Appl Mech Eng 89:141-219

40. Shariff K, Moser RD (1998) Two-dimensional mesh embedding for B-spline methods. J Comput Phys 145:471-488

41. Tezduyar TE (2003) Computation of moving boundaries and interfaces and stabilization parameters. Int J Numer Methods Fluids 43:555-575

42. Tezduyar TE, Sathe S (2004) Enhanced-discretization space-time technique (EDSTT). Comput Methods Appl Mech Eng 193:13851401

43. Tezduyar TE, Sathe S (2006) Enhanced-discretization selective stabilization procedure (EDSSP). Comput Mech 38:456-468 